\title{
Zipcode Binding Protein 1 (ZBP1; IGF2BP1): A Model for Sequence-Specific RNA Regulation
}

\author{
Jeetayu Biswas, ${ }^{1}$ Leti Nunez, ${ }^{1,5}$ Sulagna Das, ${ }^{1,5}$ Young J. Yoon, ${ }^{1,2}$ \\ Carolina Eliscovich, ${ }^{1,3}$ and Robert H. SingeR ${ }^{1,2,4}$ \\ ${ }^{1}$ Department of Anatomy and Structural Biology, ${ }^{2}$ Department of Neuroscience, ${ }^{3}$ Department of \\ Medicine, Albert Einstein College of Medicine, Bronx, New York 10461, USA \\ ${ }^{4}$ Howard Hughes Medical Institute, Janelia Research Campus, Ashburn, Virginia 20147, USA \\ Correspondence: robert.singer@einsteinmed.org
}

\begin{abstract}
The fate of an RNA, from its localization, translation, and ultimate decay, is dictated by interactions with RNA binding proteins (RBPs). $\beta$-actin mRNA has functioned as the classic example of RNA localization in eukaryotic cells. Studies of $\beta$-actin mRNA over the past three decades have allowed understanding of how RBPs, such as ZBP1 (IGF2BP1), can control both RNA localization and translational status. Here, we summarize studies of $\beta$-actin mRNA and focus on how ZBP1 serves as a model for understanding interactions between RNA and their binding protein(s). Central to the study of RNA and RBPs were technological developments that occurred along the way. We conclude with a future outlook highlighting new technologies that may be used to address still unanswered questions about RBP-mediated regulation of mRNA during its life cycle, within the cell.
\end{abstract}

Changes in protein synthesis define all aspects of a cell's life. Although most cells carry identical copy numbers of their DNA, differences in transcription and translation define both cellular fate and moment to moment cellular decisions. Therefore, the regulation of RNA is central to the control of protein synthesis (for review, see Vera et al. 2016). The timing and localization of protein synthesis is highly regulated at the level of RNA by RNA binding proteins (RBPs).

In eukaryotes, RBPs shuttle between the nucleus and cytoplasm participating in all aspects of gene expression. RBPs can direct the processes of splicing, polyadenylation, export, localization, translation, and decay (for review, see Glisovic et al. 2008). RBPs allow for rapid spatiotemporal regulation of transcripts. Although long-term changes in steady state RNA abundance can occur during differentiation, there are several instances when more rapid modulation of transcripts is necessary. By storing transcripts in a translationally inactive state until stimulated, cellular RBPs can rapidly initiate localized translation.

RNA localization patterns appear across organisms and cell types (for reviews, see Martin and Ephrussi 2009; Buxbaum et al. 2015). Asymmetric distribution of poly(A) RNAs was first described in ascidian eggs (Jeffery et al. 1983). Shortly thereafter, specific RNAs were observed to be localized to the animal and vegetal pole in maternal Xenopus eggs (Rebagliati et al. 1985). In chicken embryonic myoblasts both tritiated and biotinylated probes were used to observe cytoskeletal mRNA localization (Singer and Ward 1982; Lawrence and Singer 1986). Of the three
RNAs tested (actin, vimentin, and tubulin), actin had the most distinct localization pattern. Approximately $95 \%$ of myoblasts had a nonrandom distribution of $\beta$-actin mRNA, in which RNAs were concentrated at the cell extremities.

Key to the observation of RNA localization was the development and optimization of RNA fluorescence in situ hybridization (RNA FISH) (Singer and Ward 1982; Lawrence and Singer 1985). Improvements to both the detectors and fluorophores allowed for visualization and tracking of single RNA molecules within cells (Bertrand et al. 1998; Femino et al. 2003). Building on the advent of single-molecule RNA FISH, advances in microfluidics have allowed for multiplexed reprobing of samples and transcriptome-wide studies of RNA localization in both cells and tissues (Levsky et al. 2002; Lécuyer et al. 2007; Eng et al. 2019). By increasing the throughput of approaches to study RNA localization, it has now been possible to appreciate the breadth of RNAs that undergo this process. For example, RNA localization was found to be prevalent in the developing Drosophila embryo (Lécuyer et al. 2007). RNA FISH against 3370 genes determined that $>70 \%$ of the RNAs tested showed different patterns of localization, not only emphasizing the prevalence of RNA localization patterns but also the different mechanisms by which localization patterns are encoded. In neurons, more than one-half of the transcripts queried by RNA sequencing were localized in the neurite and nearly one-half of the localized mRNAs led to neurite localized proteins (Zappulo et al. 2017).

\footnotetext{
${ }^{5}$ These authors contributed equally to this work.

(C) 2019 Biswas et al. This article is distributed under the terms of the Creative Commons Attribution-NonCommercial License, which permits reuse and redistribution, except for commercial purposes, provided that the original author and source are credited.
} 
Profiling of RNAs from different subcellular compartments shows that RNA localization confers function on the subcellular scale. Within the cytoplasm, localization and local translation of mRNAs to the mitochondria and endoplasmic reticulum (ER) ensures correct expression of mitochondrial and secretory proteins (Garcia et al. 2010; Gadir et al. 2011; Jan et al. 2014; Williams et al. 2014). In the migrating cell, $\beta$-actin mRNA localization to the leading edge allows for directional movement in response to guidance cues (Lawrence and Singer 1986; Shestakova et al. 2001; Katz et al. 2012).

RNA transport and localization provide the means for gene expression regulation, in which local sites can determine what proteins are made in response to spatiotemporal cues. In highly polarized neurons, there are clear advantages to local control of gene expression. As dendrites and axons can extend hundreds of microns away from the cell body, the process of RNA localization and local protein synthesis is particularly relevant to neuronal function (Zappulo et al. 2017). Synapses that mediate transmission of information from one neuron to another have the capacity to undergo long-term cytoskeletal remodeling, growing or shrinking the size of the dendritic spine in response to firing (Hotulainen and Hoogenraad 2010). This synaptic modulation requires newly translated proteins and underlies higher cognitive functions such as learning and memory (for review, see Costa-Mattioli et al. 2009).

In the developing axon growth cone, RNA localization has been shown to be involved in growth cone pathfinding, allowing the axon to twist and turn as it navigates chemical gradients and forms immature synapses (Eom et al. 2003). In the mature neuron, dynamic RNA localization occurs in response to neural stimulation of specific dendrites, with RNAs localizing to their base and new protein synthesis occurring in the specifically stimulated spines (Yoon et al. 2016). Therefore, not only does the timing of translation play a key role when a dendrite is stimulated but also the proper RNA location at a specific synapse is critical.

\section{MECHANISMS OF RNA LOCALIZATION}

It is now appreciated that RNA localization, facilitated by RBPs, is a highly conserved mechanism to spatially confine protein synthesis, amplify local protein concentration, or even direct integration into macromolecular complexes (for reviews, see Glisovic et al. 2008; Buxbaum et al. 2015). Compartment-specific targeting of mRNA involves recognition of a short nucleotide sequence known as a cis-acting localization element by an RBP called the trans-acting factor. Many well-characterized RBPs bind sequence specifically, whereas others have been shown to recognize specific stem-loop structure or nonspecifically bind to single- or double-stranded RNA. The direct association between RBPs and cis-acting localization elements leads to the formation of a ribonucleoprotein (RNP) complex that travels along the cytoskeleton with the help of motor proteins (for review, see Eliscovich et al. 2013). Identification of canonical trans-acting factors have been based on (i) their ability to interact specifically with their target mRNAs, and (ii) where loss of function or loss of expression results in mislocalization of the mRNA. Disruptions in the formation of transport RNPs can have significant consequences, especially in neurons in which localization is necessary for the synthesis of proteins and consequently synaptic plasticity.

Several models have been developed to understand how RNAs can become localized within a cell (for review, see Buxbaum et al. 2015). RNA molecules can passively diffuse until they reach an anchoring point (diffusion and entrapment). Different examples of anchors include cytoskeletal proteins or RBPs (Beach et al. 1999; Farina et al. 2003). RNAs can also be locally protected from degradation or conversely selectively degraded, leading to the accumulation of RNA in areas with lower decay rates (Zaessinger et al. 2006; Tadros et al. 2007). Additionally, RNAs can be localized by motor proteins, often through their interaction with an RBP that serves as an adapter (Long et al. 2000; Song et al. 2015). Although each mechanism of localization can act alone, they can also be combined to perform biological functions. By combining directed transport with local entrapment, it has been shown that $\beta$-actin mRNA can rapidly localize to a stimulated dendritic spine where new proteins are then synthesized (Yoon et al. 2016). Key to both simple and complex modes of RNA localization are RBPs.

\section{ZBP1: THE RBP THAT CONTROLS $\beta$-ACTIN mRNA LOCALIZATION}

Since the initial observation of polarized RNA within cells, $\beta$-actin mRNA localization has provided a model system for understanding the mechanisms of RNA localization within eukaryotic cells (Fig. 1; for reviews, see Eliscovich et al. 2013; Buxbaum et al. 2015; Eliscovich and Singer 2017; Das et al. 2019). Biochemical methodologies, structural analysis, and imaging-based approaches have analyzed how a cis-acting element and transacting factors act together to ensure the cytoplasmic fate of the mRNA once it is transcribed in the nucleus.

Cytoplasmic $\beta$-actin mRNA localization was initially observed using in situ hybridization in chicken embryonic skeletal myoblasts and fibroblasts (Lawrence and Singer 1986). Later reporter plasmids expressing different regions of the 3' UTR of $\beta$-actin mRNA demonstrated that a 54-nt cis-acting "zipcode" element was responsible for the localization of $\beta$-actin mRNA to the cellular periphery (Kislauskis et al. 1994). This 54-nt zipcode sequence is conserved from chicken to mouse and human $\beta$-actin 3' UTRs. Zipcode Binding Protein 1 (ZBP1; also called IGF2BP1), the key RBP that binds the 54-nt zipcode in the nucleus and regulates localization and translational repression of $\beta$-actin mRNA in the cytoplasm (Farina et al. 2003; Oleynikov and Singer 2003; Hüttelmaier et al. 2005; Pan et al. 2007; Wu et al. 2015), was identified by UV crosslinking and affinity purification (Ross et al. 1997). Subsequently, a number of RBPs homologous to ZBP1 were also discovered in human, mouse, fly, and frog (Yisraeli 2005). A timeline highlighting ZBP's role as a critical 


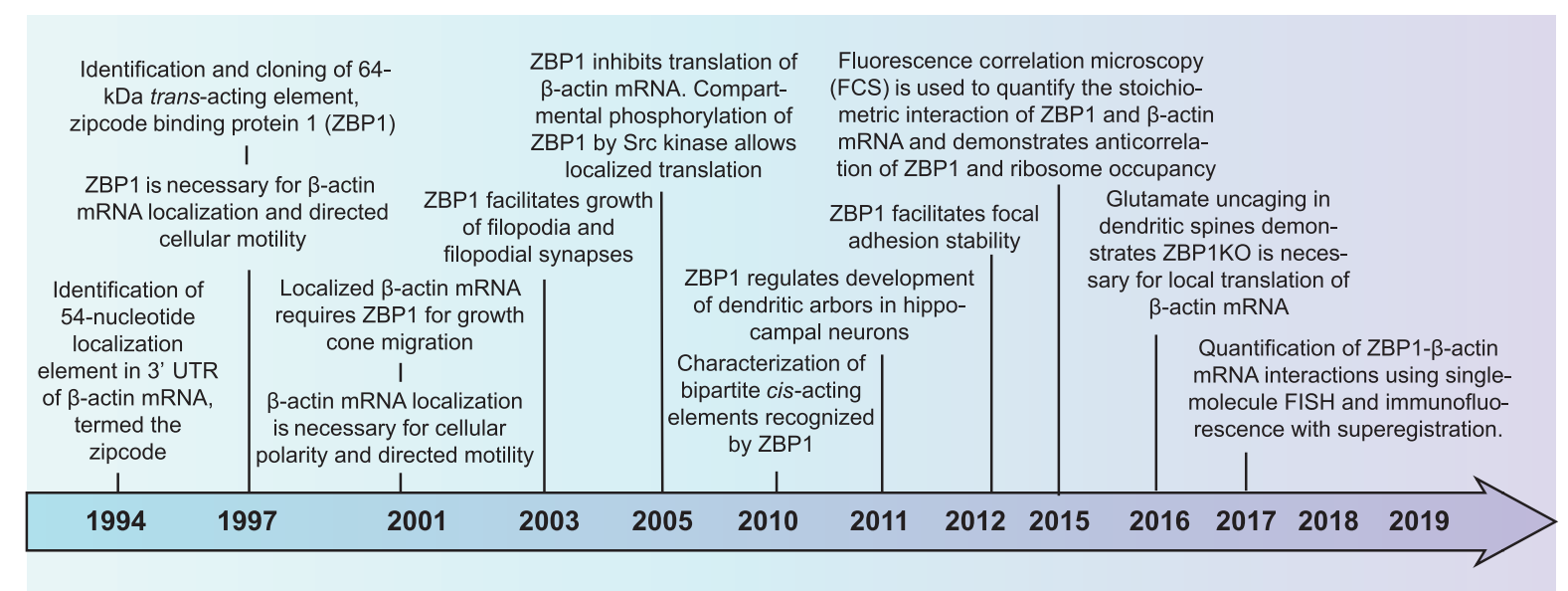

Figure 1. Timeline of ZBP1 and $\beta$-actin mRNA discoveries. Over the past two decades, significant work has revealed that the zipcode binding protein-1 (ZBP1) is involved in localization of mRNA. Presented here is a summary of important findings that have led to the current understanding of how ZBP1 acts to regulate $\beta$-actin mRNA.

RBP for $\beta$-actin mRNA localization in different cell types has been illustrated in Figure 1.

RNA reporter assays (Kislauskis et al. 1994) and biochemical (Farina et al. 2003) and structural characterization of the 54-nt zipcode led to the identification of a minimal 28-nt consensus bipartite element that is specifically recognized by ZBP1 (Chao et al. 2010; Patel et al. 2012). The structural studies also showed that ZBP1KH34 (third and fourth hnRNP K-homology domains) specifically binds the bipartite $\beta$-actin $3^{\prime}$-UTR element, with ZBP1KH4 and ZBP1KH3 recognizing $\beta$ actin 5'-CGGAC-3' and 5'-(C/A)CA(C/U)-3' sequences, respectively (Chao et al. 2010; Patel et al. 2012). For each RNA consensus sequence to bind to the two protein binding sites on opposite ends of ZBP1KH34, the RNA must loop around the protein. The distortion sequesters the stop codon of $\beta$-actin mRNA and likely contributes to translational repression (Figs. 2 and $3 \mathrm{~A}-\mathrm{D}$; Chao et al. 2010; Wu et al. 2015).

\section{ZBP1 CONTROLS LOCAL $\beta$-ACTIN TRANSLATION}

Both the zipcode sequence within the 3' UTR of $\beta$-actin and the KH34 domains of ZBP1 are necessary for the formation of the ZBP1- $\beta$-actin mRNA complex (Fig. $2 A, B)$. Regulation of $\beta$-actin mRNA fate is dependent on these associations.

In the cytoplasm, formation of the ZBP1- $\beta$-actin mRNA complex sterically inhibits the large ribosomal subunit from binding the $\beta$-actin mRNA, thereby preventing translation (Hüttelmaier et al. 2005; Wu et al. 2015). Motorized movement of this complex along cytoskeletal filaments leads to peripheral localization (e.g., leading edge in fibroblasts, dendrites or axonal cone in neurons) and accounts for the nonrandom distribution of $\beta$-actin mRNA observed in migrating or stimulated cells (Bassell et al. 1998; Mukherjee et al. 2019). Src kinase-dependent phos- phorylation of Tyr-396 in ZBP1 leads to disassembly of the ZBP1- $\beta$-actin mRNA complex at the periphery. Subsequent release of $\beta$-actin mRNA permits binding of ribosomal subunits and leads to localized translation (Fig. 2C; Hüttelmaier et al. 2005). Thus, ZBP1 can both localize and control the translation of $\beta$-actin mRNA, suggesting that RBPs can perform complex roles within the cells.

A similar pattern of events occurs in yeast when ASHI mRNA localizes to the bud tip in a She2-dependent manner (Long et al. 2000). Motor-dependent localization along cytoskeletal filaments and phosphorylation-dependent translation initiation in both yeast and mammalian cells suggests there exist common mechanisms by which RBPs can localize RNAs and regulate their translation.

Although ZBP1's regulation of $\beta$-actin mRNA is well characterized, it is likely that multiple RBPs bind to and regulate a given RNA during its entire life cycle. ZBP1 binding to the $\beta$-actin $3^{\prime}$ UTR is in coordination with other proteins, such as ZBP2 (human hnRNP protein K-homology splicing regulator protein, KHSRP). Binding of ZBP2/KHSRP in the nucleus facilitates nuclear ZBP1 association and further cytoplasmic localization in fibroblasts (Gu et al. 2002; Farina et al. 2003). Therefore, the binding of an RBP to its target can be facilitated or hindered by cofactors. Perturbations of mRNA-RBP interactions leads to functional consequences in multiple cell types. For example, in fibroblasts, the absence of the mRNA zipcode or ZBP1 results in loss of polarity, focal adhesions, and random mRNA movement (Shestakova et al. 2001; Katz et al. 2012). Similarly in neurons, loss of either the zipcode or ZBP1 affects synaptic formation and dendritic branching (Perycz et al. 2011). The neuronal observations are consistent with $\beta$-actin protein being an important cytoskeletal component involved in growth cone migration and facilitating synapse formation. Loss of the zipcode or ZBP1 in forebrain neurons is associated with decreased growth cone migration (Zhang et al. 2001; Welshhans and Bassell 2011). Hippocampal neurons, in 
A Structure

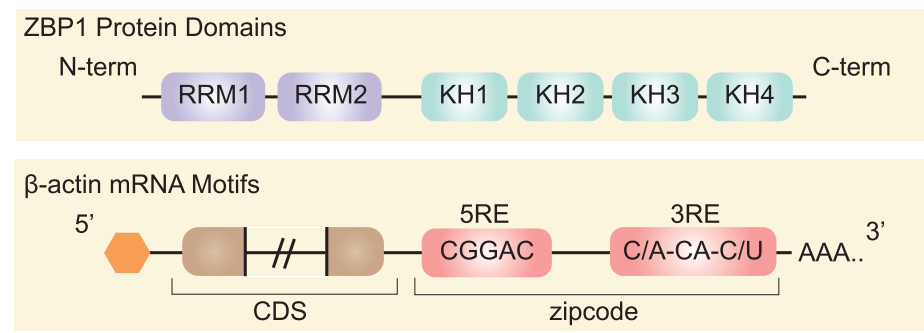

B Molecular Interaction

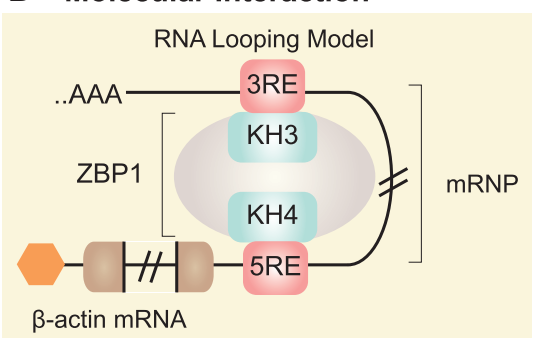

C Cellular Mechanism

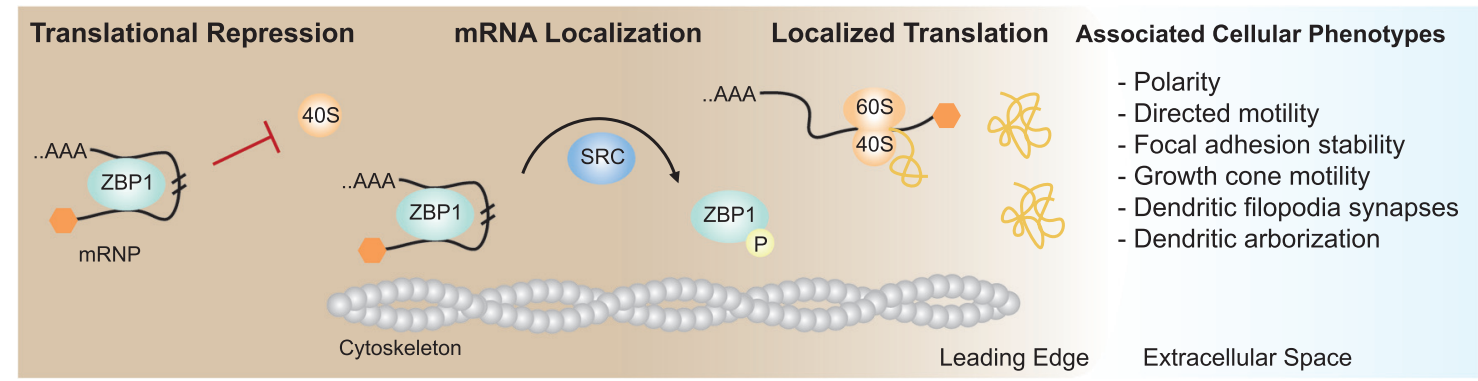

Figure 2. Summary of the molecular interaction of ZBP1 with $\beta$-actin mRNA, which determines cellular fate. $(A)$ The interaction of ZBP1 with cognate mRNA; $\beta$-actin is dependent on structural motifs. ZBP1 contains two RNA recognition motifs (RRM) (purple) and four hnRNP-K homology (KH) (green) domains. $\beta$-actin mRNA contains a bipartite localization element in the 3' UTR, named the zipcode (red). $(B)$ Interaction between the zipcode and ZBP1 has been proposed to occur by RNA looping, thereby forming the ribonucleoprotein complex (mRNP). $(C)$ Formation of the mRNP complex has molecular and cellular consequences. The ZBP1- $\beta-$ actin mRNP prevents ribosomal subunits (e.g., 40S) from binding $\beta$-actin mRNA, thus resulting in translational repression. Active transport of the ZBP1- $\beta$-actin mRNP along cytoskeletal filaments (motors not depicted) allows localization of $\beta$-actin mRNA. Disassembly of the ZBP1- $\beta$-actin mRNP is facilitated by Src-dependent phosphorylation of ZBP1 at the leading edge. Release of $\beta$-actin mRNA ultimately allows for ribosomal subunit assembly and local translation to occur. Localized translation of $\beta$-actin mRNA at the leading edge is associated with cellular phenotypes including polarity, directed motility, and focal adhesion stability in fibroblasts and growth cone motility, dendritic filopodia synapses, and dendritic arborization in neurons.

contrast, have notable changes to dendritic arborization, with a severe reduction in branching and filopodia formation (Fig. 3I; Eom et al. 2003; Perycz et al. 2011). Taken together, these studies demonstrate the importance of these conserved and coordinated molecular events underlying localized translation. However, the role of ZBP1 extends beyond translation by functioning as an adapter between the RNA and cytoskeletal motors and, thus, trafficking RNAs to their final destinations (Song et al. 2015).

\section{RNA TRANSPORT IN DENDRITES}

The dendritic arbor represents a complex maze for mRNA transport and trafficking. Real-time imaging and tracking of mRNAs allows understanding of the basic rules for transport behavior. Endogenous and reporter mRNAs containing zipcodes in their $3^{\prime}$ UTRs exhibit similar velocities during directed motion, consistent with the cis-acting element(s) and the trans-acting factor(s) being the critical determinants of RNP transport. With neuronal stimulation, the $\beta$-actin mRNP can become unmasked and release a translatable pool of $\beta$-actin mRNA in distal dendrites (Buxbaum et al. 2014). To determine whether mRNP localization and unmasking could be controlled, glutamate uncaging delivered neurotransmitter to a subset of dendritic spines. Upon localized stimulation, endogenous $\beta$-actin mRNAs localized to the stimulated synapses, resulting in an accumulation of mRNAs over 2 hours. This local capture of $\beta$-actin mRNPs was blocked in neurons from the ZBP1 knockout mice, suggesting the role of ZBP1 as the targeting protein (Fig. 3H; Yoon et al. 2016).

When the mRNP particles are in motion, they move processively $(0.5-2.0 \mu \mathrm{m} / \mathrm{sec})$, move in a series of short distances (few microns) intervened by short pauses $(<10$ $\mathrm{sec}$ ), or remain corralled (diffusion within a small volume of space). The directed movement is indicative of motordriven transport along microtubule tracks, with instantaneous velocities ranging from 0.5 to $5 \mu \mathrm{m} / \mathrm{sec}$ (Yoon et al. 2016; Das et al. 2018). Dendritic mRNPs can move in either direction or switch directions - depending on the combined force of the bound motors and the orientation of the microtubules. Collectively, all dendritic mRNAs exhibit bidirectional motion, but with a slight bias toward the anterograde, which allows them to be delivered to the distal dendrite to participate in local translation when needed.

mRNA localization in dendrites supports a "local entrapment" model as a general mechanism of how local activity can capture mRNAs that are cruising along the dendrites. This represents a "sushi belt" where $\beta$-actin mRNA(s) patrol through multiple synapses like a circling conveyor belt until they are captured by the recently activated synapses and anchored to the base of the spine (Doyle and Kiebler 2011). To determine the fate of the 
Structural Interactions of ZBP1 and $\beta$-actin mRNA

A

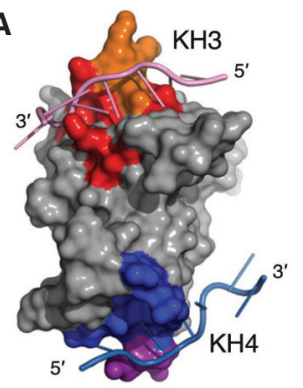

B

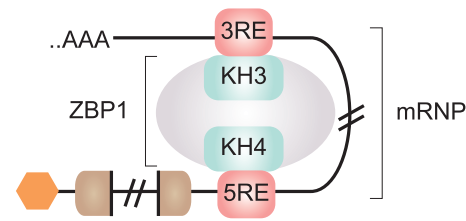

$\beta$-actin mRNA

Quantifying ZBP1 and $\beta$-actin mRNA binding

C

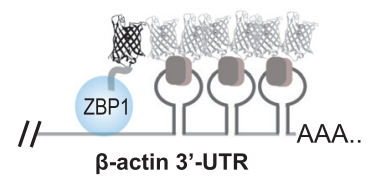

E

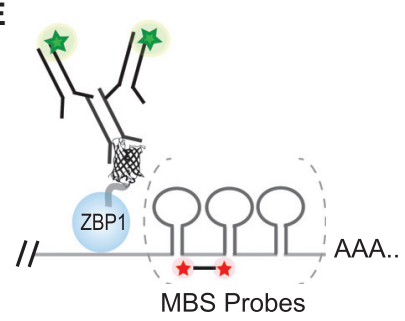

$\beta$-actin 3' UTR

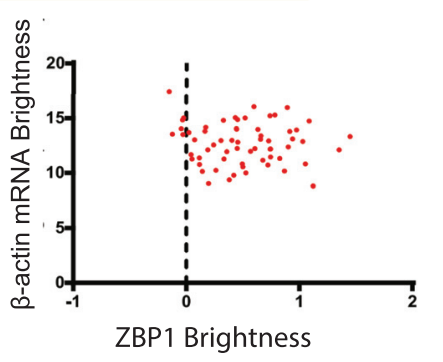

F

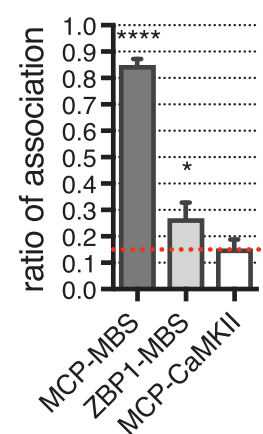

Alteration of ZBP1- $\beta$-actin mRNA Interactions Affects Cellular Phenotypes

G
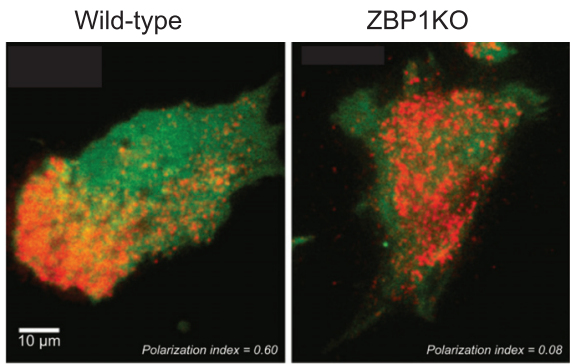

$\mathrm{H}$
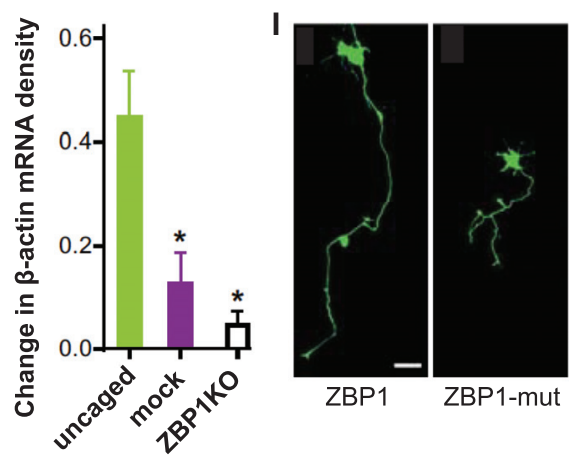

Figure 3. Exploring RBP-RNA interactions by studying ZBP1- $\beta$-actin mRNA. Here, we highlight studies that have contributed to our understanding of ZBP1- $\beta$-actin mRNA structural interactions $(A, B)$, stoichiometry of binding $(C-F)$, and how alterations of their interactions lead to respective changes in cellular phenotype $(G-I)$. ZBP1 has two main domains (KH34) that are reported to interact with the zipcode element in the $3^{\prime}$ UTR of $\beta$-actin $(A)$, which is proposed to form an RNA loop when bound by ZBP1 $(B)$. The stoichiometric interaction of ZBP1 with $\beta$-actin mRNA has revealed a 1:1 binding. Two parallel imaging approaches have been utilized to confirm the stoichiometric associations of the ZBP1- $\beta$-actin complex: fluctuation correlation microscopy (FCS) $(C, D)$ and fluorescence in situ hybridization and immunofluorescence (FISH-IF) $(E, F)$. Using the bacteriophage MS2 system to label $\beta$-actin mRNA with stem-loops (MBS), capsid proteins are labeled with fluorescent proteins. Simultaneous expression of ZBP1 with a fluorescent tag $(C)$. Using live tracking of single particles in FCS videos, the fluorescent intensity of both particles is used to evaluate stoichiometry $(D)$. Alternatively, fixed images from FISH-IF can be analyzed to determine stoichiometric association. Using a similar approach, ZBP1 is expressed with a fluorescent tag. To increase the signal from IF, staining is performed against thee fluorescent tag. FISH probes are used against the stem-loop sequence (MBS) $(E)$ and spatial association of signals are used to determine stoichiometry $(F)$. Alteration of the ZBP1- $\beta$-actin mRNA complex leads to a variety of cellular defects. In fibroblasts, deletion of ZBP1 leads to loss of polarization. In neurons, ZBP1 is important for localization of $\beta$-actin mRNA in dendrites. The localization of $\beta$-actin mRNA occurs in response to glutamate stimulation (photoactivable uncaging of glutamate). Loss of ZBP1 results in a reduction of $\beta$-actin mRNA in response to glutamate release $(H)$. Neurons also show alterations in outgrowth in response to mutations of the ZBP1 phosphorylation site (Hüttelmaier et al. 2005). (A, Modified, with permission, from Patel et al. 2012, C Cold Spring Harbor Laboratory Press; $D$, modified from Wu et al. 2015, (C) Elsevier; F, modified from Eliscovich et al. 2017; H, adapted from Yoon et al. 2016.)

localized mRNP, $\beta$-actin translation reporters showed that after an mRNA localizes to an activated spine, it persists there for hours undergoing multiple rounds of stimulation and translation to generate a pool of new $\beta$-actin proteins that can be incorporated into the expanding dendritic spine structure. Future studies are required to validate whether this "sushi belt" model of mRNA transport is applicable to other dendritically localized mRNAs along the entire length of the dendrite.

To determine the conservation of ZBP1-dependent 1ocal translation for transcripts other than $\beta$-actin, transcriptome-wide profiling of ZBP1 interacting transcripts can subsequently be evaluated for physiologically relevant interactions. 


\section{TECHNOLOGICAL PERSPECTIVES ON RBPs: GENOME- AND PROTEOME-WIDE APPROACHES}

Given the complex nature of RNA-protein interactions, it is now appreciated that few transcripts act with a single RBP, and few RBPs act upon a single transcript. To determine the breadth of interactions development of genome-wide approaches has been essential. Although each technique focuses on one aspect of RNA regulation (binding to a target, downstream effects on stability, translation regulation), the integration of multiple approaches provides the opportunity to uncover the breadth and depth of RBP-based regulation (Lapointe et al. 2018). Here we highlight a few techniques that have been used to study ZBP1 and related RBPs and hypothesize a few possible themes that may result from their integration.

To determine the targets of an RBP, the most notable approaches are RIP (RNA immunoprecipitation) and its successor CLIP (UV cross-linking immunoprecipitation) (for review, see Wheeler et al. 2018). Antibodies are used to isolate the RBP of interest, and next-generation sequencing is used to identify the fragments that are isolated. However, a number of limitations exist with this approach, the foremost being related to the indirect isolation of RBPs with antibodies. Varying degrees of antibody specificity and affinity are used, making the comparison of CLIP studies across groups, cell lines, and experiments challenging. Recently an effort lead by the ENCODE project has characterized commercial antibodies and optimized the CLIP protocol (Van Nostrand et al. 2016). With the optimized protocol, hundreds of RBPs have been studied in HepG2 and K562 cells (Van Nostrand et al. 2016). However, the process of UV cross-linking, antibody-based immunoprecipitation as well as most of the downstream processing steps have known associated biases. Complementary approaches to CLIP have been necessary to validate the putative targets that come from this approach.

One such promising in vitro approach performs highly multiplexed measurements of protein $\mathrm{ON}$ and OFF rates with thousands of randomly synthesized RNA targets. Named RNA arrays on high-throughput sequences (RNA-HiTS), this approach utilizes the microscope, microfluidics, and flow cells of DNA sequencers. Thousands of RNA sequences are synthesized during sequencing, and individual cluster locations are annotated and then interrogated by flowing in fluorescent RBPs (for review, see Denny and Greenleaf 2019). By determining the landscape of binding affinities in vitro, rules by which RBPs recognize the sequence and structures of their targets can be interrogated. This allows for refinement of CLIP and other in vivo data, motifs found by RNAHiTS can be used to identify both false positives and false negative such as putative targets that may not be highly expressed in the cell type used.

Two antibody independent approaches to study RBPs were recently developed, RNA tagging in yeast and TRIBE (targets or RNA binding proteins identified by editing) in Drosophila. Both approaches utilize enzymes fused to a RBP of interest to deposit covalent marks on
RNA targets. RNA tagging utilizes yeast genetics to endogenously fuse poly(U) polymerase (Caenorhabditis elegans PUP-2 which lacks an RNA binding domain) to the RBP of interest. " $U$ "-tailed RNAs are then isolated by poly(A) selection, after which paired-end sequencing libraries are generated. Poly(U) tail length for RNA targets in yeast correlated with both affinity for the RBP and cellular function (Lapointe et al. 2015). TRIBE fuses Drosophila adenosine deaminase (ADAR) to an RBP of interest. After FACS sorting of cells expressing the ADAR-RBP fusion protein, standard RNA sequencing library preparation is then performed. Edited RNAs are then identified by the heterogeneity of nucleotides at individual positions along a transcript (McMahon et al. 2016; Rahman et al. 2018; Xu et al. 2018).

Whereas neither RNA tagging nor TRIBE provide the nucleotide level resolution of CLIP, they may select for longer-lived RNA-protein interactions and provide methods complementary to CLIP. The recent adaptation and application of TRIBE in mammalian cells (Biswas et al. $2019 b$ ) allowed for the profiling of mammalian RBPs such as ZBP1 and its family members (J Biswas, R Rahman, V Gupta, et al., in prep.). Data comparing mammalian TRIBE to CLIP suggests that TRIBE can avoid the biases and false positives associated with antibody immunoprecipitation and allow for all RBP binding sites to be discovered within a cell (Biswas et al. 2019b; J Biswas, et al., in prep.).

To determine the proteins associated with a specific transcript, biochemical techniques such as complementary biotinylated oligonucleotide-based isolation of RNA followed by mass spectrometry have classically been used (Ross et al. 1997). Recent advances in proximitybased labeling techniques using the biotin ligases have emerged as a powerful complementary approach to map RNA-protein interactions. One of the most studied biotin ligases is BirA, a bifunctional protein expressed in Escherichia coli, which mediates biotinylation of a specific lysine residue of a subunit of acetyl-CoA carboxylase. Using a mutated BirA, which causes promiscuous biotinylation, proximity-dependent biotin identification (BioID) was developed, which labels any proximal proteins within a radius of $\sim 10 \mathrm{~nm}$ (Kim and Roux 2016). The $\beta$-actin mRNA associated proteome was identified by performing BioID upon $\beta$-actin mRNA in MEFs (Mukherjee et al. 2019). Besides ZBP1, this approach identified novel regulators of $\beta$-actin mRNA localization including FUBP3. Additionally, this approach determined which factors were constitutively loaded upon the RNA and which exchanged during the process of serum starvation or stimulation in which localization to the leading edge is decreased or increased respectively. BioID has been extended to map large-scale RNP interactome by analyzing the biotinylation profile of 119 proteins associated at different stages of the mRNA life cycle (Youn et al. 2018). However, one caveat to BioID is that it requires biotinylation over $16-24 \mathrm{~h}$ and therefore captures multiple interactions occurring over a long period of time. For more rapid labeling, TurboID (Branon et al. 2018), ascorbate peroxidase (APEX) (Lam et al. 2015), and RNA-protein interaction detection (RaPID) (Ramanathan et al. 2018) 
are the techniques of choice, which can capture transient interactions in situ, in different subcellular compartments. An engineered APEX, which oxidizes biotin-phenol, generates short-lived radicals that can covalently react with tyrosine and other electron-rich amino acids as well as the amino group on guanosine (Kim and Roux 2016). The optimization of the enzyme tags and the ability to biotinylate both RNA and proteins have extended the capability of APEX to map RNA-protein interactions in cells with subcellular precision (Kaewsapsak et al. 2017; Fazal et al. 2019). More recently, proximity biotinylation by APEX has been used to profile the RNAs associated with different organelles and stress granules (Fazal et al. 2019; Padrón et al. 2019). Future studies may allow for the profiling of RNA granule components using APEX-based strategies.

\section{IMAGING-BASED APPROACHES TO STUDY RNA-PROTEIN INTERACTIONS IN SITU}

The study of an mRNA and its binding protein(s) by ensemble biochemical approaches (Mili and Steitz 2004) lacks spatial information. Given the wealth of RNA-protein interactions now defined by the aforementioned techniques, follow-up experiments are required to determine in situ RNA-protein interactions as well as subcellular RNA localization. Imaging-based approaches therefore can provide spatial information currently inaccessible by other methods, and this is particularly critical for the understanding of RNA localization.

High-precision imaging-namely, "super registration"- is capable of determining whether two molecules are physically interacting in situ or simply in proximity by random chance using standard wide-field microscopy (Fig. 3E,F; Eliscovich et al. 2017). This imaging technique corrects the chromatic aberration uniquely intrinsic to individual commercial microscope objectives so that two molecules can be superimposed within $10 \mathrm{~nm}$ precision. By precisely localizing the mRNA and protein using single-molecule FISH to detect mRNA and immunofluorescence (IF) against a RBP, a significant fraction of proteins biochemically defined to bind $\beta$-actin mRNA were then shown to not interact with the RNA in situ. Therefore, super registration can complement information from biochemical interactions and visualize physical contacts in situ, with high precision in both neurons and cell lines (Eliscovich et al. 2017; Mukherjee et al. 2019).

Direct RNA-protein interactions within living cells can be interrogated with multicolor fluorescence correlation spectroscopy. By exciting a femtomolar volume within the cell, the association of different molecules can be correlated over time at a specific point in space. This approach can be used alongside super registration microscopy to determine live cell associations of RNA (labeled with an MBS array) with fluorescently tagged RBPs (Fig. 3C,D; $\mathrm{Wu}$ et al. 2015). Future developments may combine this approach with super-resolution STED imaging (Lanzanò et al. 2017) to allow for interactions to be measured within a dense environment - for instance, in an individual dendritic spine.

Although single-molecule imaging often interrogates individual genes or proteins, recent advances in highly multiplexed RNA FISH can now localize thousands of RNA species inside the cell. By using iterative RNA FISH and multiplex barcoding of the FISH probes, techniques such as SeqFISH (Eng et al. 2019) and MERFISH (Xia et al. 2019) allow transcriptome-wide localization of RNAs within both cells and tissues. These approaches have found novel transcripts localized to cellular protrusions, expanding our understanding of RNA localization (Eng et al. 2019). Future applications of these techniques may follow the entire breadth of ZBP1 targets (as defined by CLIP or TRIBE) in rapidly changing environments such as the migrating fibroblast, developing growth cone, or stimulated synapse. Although both approaches have been combined with single color IF, recent multicolored fluorescence imaging of barcoded antibodies has allowed for spatial profiling of more than a dozen different proteins (Guo et al. 2019). Combined barcoding of RNA and protein may allow for future colocalization of the two molecules at a massively parallel scale within cells.

Instead of using oligonucleotide barcoding, other approaches have performed direct sequencing of RNAs in situ. Approaches such as in situ transcriptome accessibility sequencing have begun to correlate in situ sequencing truncation events with the location of RBP binding sites. Using this approach, it was discovered that Drosophila ZBP1 (dIMP1) binding sites were found within the Act5C mRNA more localized to the periphery of retinal cells (Fürth et al. 2019). As evidenced by the above technologies, further development of high-throughput protein and RNA imaging will allow for RNA-protein interactions to be directly imaged in situ at a massively parallel scale.

\section{CONCLUSIONS-BIOLOGICAL PERSPECTIVES ON RBPS}

As evidenced above, work on $\beta$-actin-ZBP1 interactions has developed models for how, when, and where RNAs become localized inside a cell. Future technical developments are required to allow the dimensions of RNA tracking to be expanded to include the entire life of an RNA as well as the entirety of its protein interactions. Tracking of RNA fate from transcription to decay is possible with the MS2 system (Tutucci et al. 2018), and high-throughput methods allow a plethora of RBP interactions to be profiled. Missing from the current studies are in vivo dynamics of RNA-protein interactions - how are regulatory factors exchanged during a RNA's lifetime? Genomewide studies like CLIP show that many RBPs interact with the same transcript, often at the same site. How does this multitude of interactions direct the fate of the transcript? Is there functional redundancy among RBP family members or RBPs from different families (Conway et al. 2016; Biswas et al. 2019a)? How do the targets of an RBP change as cellular identity changes during differentiation or reprogramming? Studies have yet to clearly 
define how changes in RBP expression or stoichiometry affect the process of finding RNA targets. These open questions will require high-throughput, time-resolved, nondestructive measurements of RNA-protein interactions, ideally within single cells.

\section{ACKNOWLEDGMENTS}

We thank members of the Singer lab past and present for their helpful discussions and contributions to the work presented in this perspective. J.B. and L.N. were supported with funding from a Medical Scientist Training Program (MSTP) Training Grant T32GM007288. J.B. was additionally supported by predoctoral fellowship F30CA214009. C.E., Y.J.Y., and R.H.S. were supported by the National Institutes of Health (NIH) grant NS083085. Y.J.Y. was also supported by NIH grant MH120496 and R.H.S by NIH grant U01DA047729.

\section{REFERENCES}

Bassell GJ, Zhang H, Byrd AL, Femino AM, Singer RH, Taneja KL, Lifshitz LM, Herman IM, Kosik KS. 1998. Sorting of $\beta$-actin mRNA and protein to neurites and growth cones in culture. J Neurosci 18: 251-265. doi:10.1523/JNEUROSCI .18-01-00251.1998

Beach DL, Salmon ED, Bloom K. 1999. Localization and anchoring of mRNA in budding yeast. Curr Biol 9: 569-578. doi:10.1016/S0960-9822(99)80260-7

Bertrand E, Chartrand P, Schaefer M, Shenoy SM, Singer RH, Long RM. 1998. Localization of ASH1 mRNA particles in living yeast. Mol Cell 2: 437-445. doi:10.1016/\$1097-2765 (00)80143-4

Biswas J, Patel VL, Bhaskar V, Chao JA, Singer RH, Eliscovich C. 2019a. The structural basis for RNA selectivity by the IMP family of RNA-binding proteins. Nat Commun 10: 4440. doi:10.1038/s41467-019-12193-7

Biswas J, Rahman R, Gupta V, Rosbash M, Singer RH. 2019b. MS2-TRIBE evaluates protein-RNA interactions and nuclear organization of transcription by RNA editing. bioRxiv doi:10 $.1101 / 829606$

Branon TC, Bosch JA, Sanchez AD, Udeshi ND, Svinkina T, Carr SA, Feldman JL, Perrimon N, Ting AY. 2018. Efficient proximity labeling in living cells and organisms with TurboID. Nat Biotechnol 36: 880-887. doi:10.1038/nbt.4201

Buxbaum AR, Wu B, Singer RH. 2014. Single $\beta$-actin mRNA detection in neurons reveals a mechanism for regulating its translatability. Science 343: 419-422. doi:10.1126/science .1242939

Buxbaum AR, Haimovich G, Singer RH. 2015. In the right place at the right time: visualizing and understanding mRNA localization. Nat Rev Mol Cell Biol 16: 95-109. doi:10.1038/ nrm3918

Chao JA, Patskovsky Y, Patel V, Levy M, Almo SC, Singer RH. 2010. ZBP1 recognition of $\beta$-actin zipcode induces RNA looping. Genes Dev 24: 148-158. doi:10.1101/gad.1862910

Conway AE, Van Nostrand EL, Pratt GA, Aigner S, Wilbert ML, Sundararaman B, Freese P, Lambert NJ, Sathe S, Liang TY, et al. 2016. Enhanced CLIP uncovers IMP protein-RNA targets in human pluripotent stem cells important for cell adhesion and survival. Cell Rep 15: 666-679. doi:10.1016/j.celrep .2016 .03 .052

Costa-Mattioli M, Sossin WS, Klann E, Sonenberg N. 2009. Translational control of long-lasting synaptic plasticity and memory. Neuron 61: 10-26. doi:10.1016/j.neuron.2008.10 .055

Das S, Moon HC, Singer RH, Park HY. 2018. A transgenic mouse for imaging activity-dependent dynamics of endoge- nous Arc mRNA in live neurons. Sci Adv 4: eaar3448. doi:10 $.1126 /$ sciadv.aar3448

Das S, Singer RH, Yoon YJ. 2019. The travels of mRNAs in neurons: do they know where they are going? Curr Opin Neurobiol 57: 110-116. doi:10.1016/j.conb.2019.01.016

Denny SK, Greenleaf WJ. 2019. Linking RNA sequence, structure, and function on massively parallel high-throughput sequencers. Cold Spring Harb Perspect Biol 11: a032300. doi:10.1101/cshperspect.a032300

Doyle M, Kiebler MA. 2011. Mechanisms of dendritic mRNA transport and its role in synaptic tagging: mechanisms of dendritic mRNA transport. EMBO J 30: 3540-3552. doi:10.1038/ emboj.2011.278

Eliscovich C, Singer RH. 2017. RNP transport in cell biology: the long and winding road. Curr Opin Cell Biol 45: 38-46. doi:10.1016/j.ceb.2017.02.008

Eliscovich C, Buxbaum AR, Katz ZB, Singer RH. 2013. mRNA on the move: the road to its biological destiny. J Biol Chem 288: 20361-20368. doi:10.1074/jbc.R113.452094

Eliscovich C, Shenoy SM, Singer RH. 2017. Imaging mRNA and protein interactions within neurons. Proc Natl Acad Sci 114: E1875-E1884. doi:10.1073/pnas.1621440114

Eng C-HL, Lawson M, Zhu Q, Dries R, Koulena N, Takei Y, Yun J, Cronin C, Karp C, Yuan G-C, et al. 2019. Transcriptomescale super-resolved imaging in tissues by RNA seqFISH. Nature 568: 235-239. doi:10.1038/s41586-019-1049-y

Eom T, Antar LN, Singer RH, Bassell GJ. 2003. Localization of a $\beta$-actin messenger ribonucleoprotein complex with zipcodebinding protein modulates the density of dendritic filopodia and filopodial synapses. J Neurosci 23: 10433-10444. doi:10 .1523/JNEUROSCI.23-32-10433.2003

Farina KL, Hüttelmaier S, Musunuru K, Darnell R, Singer RH. 2003. Two ZBP1 KH domains facilitate $\beta$-actin mRNA localization, granule formation, and cytoskeletal attachment. J Cell Biol 160: 77-87. doi: $10.1083 /$ jcb.200206003

Fazal FM, Han S, Parker KR, Kaewsapsak P, Xu J, Boettiger AN, Chang HY, Ting AY. 2019. Atlas of subcellular RNA localization revealed by APEX-Seq. Cell 178: 473-490.e26. doi:10 $.1016 /$ j.cell.2019.05.027

Femino AM, Fogarty K, Lifshitz LM, Carrington W, Singer RH. 2003. Visualization of single molecules of mRNA in situ. Methods Enzymol 361: 245-304. doi:10.1016/S0076-6879 (03)61015-3

Fürth D, Hatini V, Lee JH. 2019. In situ transcriptome accessibility sequencing (INSTA-seq). bioRxiv doi:10.1101/722819

Gadir N, Haim-Vilmovsky L, Kraut-Cohen J, Gerst JE. 2011. Localization of mRNAs coding for mitochondrial proteins in the yeast Saccharomyces cerevisiae. RNA 17: 1551-1565. doi:10.1261/rna.2621111

Garcia M, Delaveau T, Goussard S, Jacq C. 2010. Mitochondrial presequence and open reading frame mediate asymmetric localization of messenger RNA. EMBO Rep 11: 285-291. doi:10.1038/embor.2010.17

Glisovic T, Bachorik JL, Yong J, Dreyfuss G. 2008. RNA-binding proteins and post-transcriptional gene regulation. FEBS Lett 582: 1977-1986. doi:10.1016/j.febslet.2008.03.004

Gu W, Pan F, Zhang H, Bassell GJ, Singer RH. 2002. A predominantly nuclear protein affecting cytoplasmic localization of $\beta$-actin mRNA in fibroblasts and neurons. J Cell Biol 156: 41-52. doi:10.1083/jcb.200105133

Guo S-M, Veneziano R, Gordonov S, Li L, Danielson E, de Arce KP, Park D, Kulesa AB, Wamhoff E-C, Blainey PC, et al. 2019. Multiplexed and high-throughput neuronal fluorescence imaging with diffusible probes. Nat Commun 10: 4377. doi:10 $.1038 / \mathrm{s} 41467-019-12372-6$

Hotulainen P, Hoogenraad CC. 2010. Actin in dendritic spines: connecting dynamics to function. J Cell Biol 189: 619-629. doi: $10.1083 /$ jcb. 201003008

Hüttelmaier S, Zenklusen D, Lederer M, Dictenberg J, Lorenz M, Meng X, Bassell GJ, Condeelis J, Singer RH. 2005. Spatial regulation of $\beta$-actin translation by Src-dependent phosphorylation of ZBP1. Nature 438: 512-515. doi:10 .1038 /nature04115 
Jan CH, Williams CC, Weissman JS. 2014. Principles of ER cotranslational translocation revealed by proximity-specific ribosome profiling. Science 346: 1257521. doi:10.1126/science .1257521

Jeffery WR, Tomlinson CR, Brodeur RD. 1983. Localization of actin messenger RNA during early ascidian development. Dev Biol 99: 408-417. doi:10.1016/0012-1606(83)90290-7

Kaewsapsak P, Shechner DM, Mallard W, Rinn JL, Ting AY. 2017. Live-cell mapping of organelle-associated RNAs via proximity biotinylation combined with protein-RNA crosslinking. Elife 6: e29224. doi:10.7554/eLife.29224

Katz ZB, Wells AL, Park HY, Wu B, Shenoy SM, Singer RH. 2012. $\beta$-Actin mRNA compartmentalization enhances focal adhesion stability and directs cell migration. Genes Dev 26: 1885-1890. doi:10.1101/gad.190413.112

Kim DI, Roux KJ. 2016. Filling the void: proximity-based labeling of proteins in living cells. Trends Cell Biol 26: 804-817. doi:10.1016/j.tcb.2016.09.004

Kislauskis EH, Zhu X, Singer RH. 1994. Sequences responsible for intracellular localization of $\beta$-actin messenger RNA also affect cell phenotype. J Cell Biol 127: 441-451. doi:10.1083/ jcb.127.2.441

Lam SS, Martell JD, Kamer KJ, Deerinck TJ, Ellisman MH, Mootha VK, Ting AY. 2015. Directed evolution of APEX2 for electron microscopy and proximity labeling. Nat Methods 12: 51-54. doi:10.1038/nmeth.3179

Lanzanò L, Scipioni L, Di Bona M, Bianchini P, Bizzarri R, Cardarelli F, Diaspro A, Vicidomini G. 2017. Measurement of nanoscale three-dimensional diffusion in the interior of living cells by STED-FCS. Nat Commun 8: 1-10. doi:10.1038/ s41467-017-00117-2

Lapointe CP, Wilinski D, Saunders HAJ, Wickens M. 2015. Protein-RNA networks revealed through covalent RNA marks. Nat Methods 12: 1163-1170. doi:10.1038/nmeth.3651

Lapointe CP, Stefely JA, Jochem A, Hutchins PD, Wilson GM, Kwiecien NW, Coon JJ, Wickens M, Pagliarini DJ. 2018. Multi-omics reveal specific targets of the RNA-binding protein Puf3p and its orchestration of mitochondrial biogenesis. Cell Syst 6: 125-135.e6. doi:10.1016/j.cels.2017.11.012

Lawrence JB, Singer RH. 1985. Quantitative analysis of in situ hybridization methods for the detection of actin gene expression. Nucleic Acids Res 13: 1777-1799. doi:10.1093/nar/13.5 .1777

Lawrence JB, Singer RH. 1986. Intracellular localization of messenger RNAs for cytoskeletal proteins. Cell 45: 407-415. doi:10.1016/0092-8674(86)90326-0

Lécuyer E, Yoshida H, Parthasarathy N, Alm C, Babak T, Cerovina T, Hughes TR, Tomancak P, Krause HM. 2007. Global analysis of mRNA localization reveals a prominent role in organizing cellular architecture and function. Cell 131: 174 187. doi:10.1016/j.cell.2007.08.003

Levsky JM, Shenoy SM, Pezo RC, Singer RH. 2002. Single-cell gene expression profiling. Science 297: 836-840. doi:10 $.1126 /$ science. 1072241

Long RM, Gu W, Lorimer E, Singer RH, Chartrand P. 2000. She $2 p$ is a novel RNA-binding protein that recruits the Myo4p-She3p complex to ASHI mRNA. EMBO J 19: 6592-6601. doi:10.1093/emboj/19.23.6592

Martin KC, Ephrussi A. 2009. mRNA localization: gene expression in the spatial dimension. Cell 136: 719-730. doi:10.1016/ j.cell.2009.01.044

McMahon AC, Rahman R, Jin H, Shen JL, Fieldsend A, Luo W, Rosbash M. 2016. TRIBE: hijacking an RNA-editing enzyme to identify cell-specific targets of RNA-binding proteins. Cell 165: 742-753. doi:10.1016/j.cell.2016.03.007

Mili S, Steitz JA. 2004. Evidence for reassociation of RNAbinding proteins after cell lysis: implications for the interpretation of immunoprecipitation analyses. RNA 10: 1692-1694. doi:10.1261/rna.7151404

Mukherjee J, Hermesh O, Eliscovich C, Nalpas N, Franz-Wachtel M, Maček B, Jansen R-P. 2019. $\beta$-actin mRNA interactome mapping by proximity biotinylation. Proc Natl Acad Sci 116: 12863-12872. doi:10.1073/pnas.1820737116
Oleynikov Y, Singer RH. 2003. Real-time visualization of ZBP1 association with $\beta$-actin mRNA during transcription and localization. Curr Biol 13: 199-207. doi:10.1016/S0960-9822(03) 00044-7

Padrón A, Iwasaki S, Ingolia NT. 2019. Proximity RNA labeling by APEX-seq reveals the organization of translation initiation complexes and repressive RNA granules. Mol Cell 75: 875887.e5. doi:10.1016/j.molcel.2019.07.030

Pan F, Hüttelmaier S, Singer RH, Gu W. 2007. ZBP2 facilitates binding of ZBP1 to $\beta$-actin mRNA during transcription. Mol Cell Biol 27: 8340-8351. doi:10.1128/MCB.00972-07

Patel VL, Mitra S, Harris R, Buxbaum AR, Lionnet T, Brenowitz M, Girvin M, Levy M, Almo SC, Singer RH, et al. 2012. Spatial arrangement of an RNA zipcode identifies mRNAs under post-transcriptional control. Genes Dev 26: 43-53. doi:10.1101/gad.177428.111

Perycz M, Urbanska AS, Krawczyk PS, Parobczak K, Jaworski J. 2011. Zipcode binding protein 1 regulates the development of dendritic arbors in hippocampal neurons. J Neurosci 31: 5271-5285. doi:10.1523/JNEUROSCI.2387-10.2011

Rahman R, Xu W, Jin H, Rosbash M. 2018. Identification of RNA-binding protein targets with HyperTRIBE. Nat Protoc 13: 1829. doi:10.1038/s41596-018-0020-y

Ramanathan M, Majzoub K, Rao DS, Neela PH, Zarnegar BJ, Mondal S, Roth JG, Gai H, Kovalski JR, Siprashvili Z, et al. 2018. RNA-protein interaction detection in living cells. Nat Methods 15: 207-212. doi:10.1038/nmeth.4601

Rebagliati MR, Weeks DL, Harvey RP, Melton DA. 1985. Identification and cloning of localized maternal RNAs from Xenopus eggs. Cell 42: 769-777. doi:10.1016/0092-8674(85) 90273-9

Ross AF, Oleynikov Y, Kislauskis EH, Taneja KL, Singer RH. 1997. Characterization of a $\beta$-actin mRNA zipcode-binding protein. Mol Cell Biol 17: 2158-2165. doi:10.1128/MCB.17 .4 .2158

Shestakova EA, Singer RH, Condeelis J. 2001. The physiological significance of $\beta$-actin mRNA localization in determining cell polarity and directional motility. Proc Natl Acad Sci 98: 7045 7050. doi:10.1073/pnas.121146098

Singer RH, Ward DC. 1982. Actin gene expression visualized in chicken muscle tissue culture by using in situ hybridization with a biotinated nucleotide analog. Proc Natl Acad Sci 79: 7331-7335. doi:10.1073/pnas.79.23.7331

Song T, Zheng Y, Wang Y, Katz Z, Liu X, Chen S, Singer RH, Gu W. 2015. Specific interaction of KIF11 with ZBP1 regulates the transport of $\beta$-actin mRNA and cell motility. J Cell Sci 128: 1001-1010. doi:10.1242/jcs. 161679

Tadros W, Goldman AL, Babak T, Menzies F, Vardy L, OrrWeaver T, Hughes TR, Westwood JT, Smibert CA, Lipshitz HD. 2007. SMAUG is a major regulator of maternal mRNA destabilization in Drosophila and its translation is activated by the PAN GU kinase. Dev Cell 12: 143-155. doi:10.1016/j .devcel.2006.10.005

Tutucci E, Vera M, Biswas J, Garcia J, Parker R, Singer RH. 2018. An improved MS2 system for accurate reporting of the mRNA life cycle. Nat Methods 15: 81-89. doi:10.1038/nmeth .4502

Van Nostrand EL, Pratt GA, Shishkin AA, Gelboin-Burkhart C, Fang MY, Sundararaman B, Blue SM, Nguyen TB, Surka C, Elkins K, et al. 2016. Robust transcriptome-wide discovery of RNA-binding protein binding sites with enhanced CLIP (eCLIP). Nat Methods 13: 508-514. doi:10.1038/nmeth.3810

Vera M, Biswas J, Senecal A, Singer RH, Park HY. 2016. Singlecell and single-molecule analysis of gene expression regulation. Annu Rev Genet 50: 267-291. doi:10.1146/annurev-ge net-120215-034854

Welshhans K, Bassell GJ. 2011. Netrin-1-induced local $\beta$-actin synthesis and growth cone guidance requires zipcode binding protein 1. J Neurosci 31: 9800-9813. doi:10.1523/JNEURO SCI.0166-11.2011

Wheeler EC, Nostrand ELV, Yeo GW. 2018. Advances and challenges in the detection of transcriptome-wide protein-RNA 
interactions. Wiley Interdiscip Rev RNA 9: e1436. doi:10.1002/ wrna. 1436

Williams CC, Jan CH, Weissman JS. 2014. Targeting and plasticity of mitochondrial proteins revealed by proximity-specific ribosome profiling. Science 346: 748-751. doi:10.1126/sci ence. 1257522

Wu B, Buxbaum AR, Katz ZB, Yoon YJ, Singer RH. 2015. Quantifying protein-mRNA interactions in single live cells. Cell 162: 211-220. doi:10.1016/j.cell.2015.05.054

Xia C, Fan J, Emanuel G, Hao J, Zhuang X. 2019. Spatial transcriptome profiling by MERFISH reveals subcellular RNA compartmentalization and cell cycle-dependent gene expression. Proc Natl Acad Sci 116: 19490-19499. doi:10.1073/pnas .1912459116

Xu W, Rahman R, Rosbash M. 2018. Mechanistic implications of enhanced editing by a HyperTRIBE RNA-binding protein. RNA 24: 173-182. doi:10.1261/rna.064691.117

Yisraeli JK. 2005. VICKZ proteins: a multi-talented family of regulatory RNA-binding proteins. Biol Cell 97: 87-96. doi:10 $.1042 / \mathrm{BC} 20040151$

Yoon YJ, Wu B, Buxbaum AR, Das S, Tsai A, English BP, Grimm JB, Lavis LD, Singer RH. 2016. Glutamate-induced
RNA localization and translation in neurons. Proc Natl Acad Sci 113: E6877-E6886. doi:10.1073/pnas.1614267113

Youn J-Y, Dunham WH, Hong SJ, Knight JDR, Bashkurov M, Chen GI, Bagci H, Rathod B, MacLeod G, Eng SWM, et al. 2018. High-density proximity mapping reveals the subcellular organization of mRNA-associated granules and bodies. $\mathrm{Mol}$ Cell 69: 517-532.e11. doi:10.1016/j.molcel.2017.12.020

Zaessinger S, Busseau I, Simonelig M. 2006. Oskar allows nanos mRNA translation in Drosophila embryos by preventing its deadenylation by Smaug/CCR4. Development 133: 45734583. doi: $10.1242 /$ dev.02649

Zappulo A, van den Bruck D, Mattioli CC, Franke V, Imami K, McShane E, Moreno-Estelles M, Calviello L, Filipchyk A, Peguero-Sanchez E, et al. 2017. RNA localization is a key determinant of neurite-enriched proteome. Nat Commun 8: 583. doi:10.1038/s41467-017-00690-6

Zhang HL, Eom T, Oleynikov Y, Shenoy SM, Liebelt DA, Dictenberg JB, Singer RH, Bassell GJ. 2001. Neurotrophininduced transport of a $\beta$-actin mRNP complex increases $\beta$ actin levels and stimulates growth cone motility. Neuron 31: 261-275. doi:10.1016/S0896-6273(01)00357-9 


\section{$\$_{\text {CSH }}^{\infty}$ Cold Spring Harbor Symposia SYMPOSIA on Quantitative Biology}

\section{Zipcode Binding Protein 1 (ZBP1; IGF2BP1): A Model for Sequence-Specific RNA Regulation}

Jeetayu Biswas, Leti Nunez, Sulagna Das, et al.

Cold Spring Harb Symp Quant Biol published online February 21, 2020

Access the most recent version at doi:10.1101/sqb.2019.84.039396

$\mathbf{P}<\mathbf{P} \quad$ Published online February 21, 2020 in advance of the print journal.

Creative This article is distributed under the terms of the

Commons http://creativecommons.org/licenses/by-nc/4.0/, which permits reuse and

License redistribution, except for commercial purposes, provided that the original author and source are credited.

Email Alerting Receive free email alerts when new articles cite this article - sign up in Service the box at the top right corner of the article or click here.

Advance online articles have been peer reviewed and accepted for publication but have not yet appeared in the paper journal (edited, typeset versions may be posted when available prior to final publication). Advance online articles are citable and establish publication priority; they are indexed by PubMed from initial publication. Citations to Advance online articles must include the digital object identifier (DOIs) and date of initial publication.

To subscribe to Cold Spring Harbor Symposia on Quantitative Biology go to: http://symposium.cshlp.org/subscriptions 